\title{
EDITORIAL
}

\section{The cellular impacts of diesel exhaust particles: beyond inflammation and death}

\author{
N. Li and A.E. Nel
}

담 pidemiological studies have demonstrated an association between exposure to ambient particulate matter (PM) and increasing respiratory mortality and morbidity [1]. In western countries, exhausts from diesel and gasoline vehicles constitute a major portion of PM and have been suspected of increasing the risk of lung cancer [2]. An important component of PM is the diesel exhaust particle (DEP), which is comprised of a carbon core that adsorbs a mixture of metals and organic chemicals, including carcinogens [3]. The small size and large surface area of DEP allow these particles to penetrate deep into the lung and deposit toxic chemicals throughout the respiratory tract [2]. Due to their location and function, epithelial cells are one of the first and major PM targets. The impact of DEP on airway epithelial cells includes inflammation, cytotoxicity and genotoxicity.

It has been established that DEP chemicals can generate reactive oxygen species (ROS) [4]. The sources of ROS generation are related to the particle content of transition metals and organic chemicals [4]. Excessive ROS can lead to a state of oxidative stress, which can induce a variety of cellular responses including DNA damage [4]. Moreover, many polycyclic aromatic hydrocarbons (PAH) associated with DEP are genotoxic as they form a PAH-DNA adduct $[5,6]$. PM-induced DNA damage includes: increased frequency of mutation; single-strand breaks; and the formation of 8-oxo-7,8dihydro-2'-deoxyguanosine and PAH-DNA adducts [2, 4].

Airway epithelial cells are at greater risk of DNA damage because of their constant exposure to PM-induced oxidative stress and carcinogens [4, 7]. In the presence of DNA damage, a control mechanism that induces cell cycle arrest is activated to ensure the fidelity of DNA replication and genomic integrity [7]. This control is exerted at the cell cycle check point in the $G_{1}$ phase [7-9]. At this phase, cells have to make the decision whether to complete another round of cell division or to exit cell cycle and arrest at the $G_{0}$ phase. Cell cycle progression is controlled by sequential assembly of cyclins and cyclindependent kinases (CDK). In the absence of mitogens, cells remain quiescent in the $G_{0}$ phase, but activate cyclin D1 expression in a ras-dependent fashion in the presence of

Dept of Medicine, Division of Clinical Immunology and Allergy, University of California, Los Angeles CA 90095, USA.

CORRESPONDENCE: A.E. Nel, Dept of Medicine, Division of Clinical Immunology and Allergy, University of California, Los Angeles, CA 90095, USA. Fax: 1 3102068107. E-mail: ANel@mednet.ucla.edu mitogens. The newly synthesised cyclin D1 forms a complex with CDK4 and CDK6, which phosphorylates the retinoblastoma gene product $(\mathrm{Rb})$ in the nucleus. Phosphorylated $\mathrm{Rb}$ dissociates from transcription factor E2F, enabling it to activate genes involved in DNA replication and cyclin E synthesis. The formation of the cyclin E-CDK2 complex shifts the cell cycle from mitogen-dependent to mitogen-independent progression $[7,8]$. In addition to the role of the cyclins, the tumour suppressor gene p53 is responsible for controlling the $G_{1}$ check point and can also induce apoptosis in response to DNA damage $[7,8]$. One of the downstream targets of p53 is p21 ${ }^{\mathrm{CIP} 1}$ /

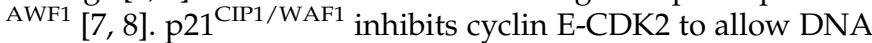
repair $[7,8]$, and is redox sensitive $[7,8,10]$. O'REILLY et al. [10] have reported a dramatic increase of $\mathrm{p} 21^{\mathrm{CIP} 1 / \mathrm{WAF} 1}$ in lung epithelial cells during hyperoxic lung injury. It is also important to mention that $\mathrm{p} 21^{\mathrm{CIP} 1 / \mathrm{WAF} 1}$ is regulated by several p53-independent mechanisms [8].

Since the 1980s, studies of DEP have mainly focused on the mechanisms by which it induces pro-inflammatory and cytotoxic effects. BAYRAM et al. [11] took a novel approach by studying the impact of DEP not only on apoptosis, but also on lung epithelial cell proliferation at the level of cell cycle regulation. Their work, which appears in this issue of the Journal [11], demonstrates that low concentrations of DEP reverse serum starvation-induced $\mathrm{G}_{0} / \mathrm{G}_{1}$ arrest in human lung epithelial cells (A549), resulting in an increase in cell numbers in DEP-treated groups. DEP also inhibited serum starvationinduced apoptosis. The enhancing effect of DEP on cell cycle progression was mediated by oxidative stress, mitogenactivated protein kinase (MAPK) and nuclear factor (NF)- $\kappa B$ signalling pathways, and cell cycle regulator $\mathrm{p} 21^{\mathrm{CIP} 1 / \mathrm{WAF} 1}$. The involvement of oxidative stress is suggested by the ability of two antioxidants, $N$-acetylcysteine and AEOL10113, to limit the increase in cell number. Using MAPK and NF- $\kappa B$ inhibitors, it is also demonstrated that DEP-induced cellular proliferation involves MAPK and NF- $\kappa \mathrm{B}$ signalling pathways. The most interesting finding of their work is that DEP inhibits serum starvation-induced $\mathrm{p} 21^{\mathrm{CIP} 1 / \mathrm{WAF} 1}$ expression. This is the first study that links the effects of DEP to p $21^{\mathrm{CIP} 1 / \mathrm{WAF} 1}$, an important redox-sensitive cell cycle regulator. This observation is particularly important when studying the association between air pollution and increased risk of lung cancer, as it has been established that PM exerts its adverse health effects via the generation of oxidative stress [4]. The results presented by BAYRAM et al. [11] suggest that interference of p21 ${ }^{\mathrm{CIP} 1 / \mathrm{WAF} 1}$ expression by DEP-induced oxidative stress may be one of the mechanisms by which long-term PM exposure induces chronic 
respiratory diseases and lung carcinogenesis. In addition, this study also suggests a new direction for future work on PM, namely the impact of PM-generated oxidative stress on oncogenes, tumour suppressor genes and cell cycle regulation.

The study also raises some important questions. The first is whether DEP-induced inhibition of $\mathrm{p} 21^{\mathrm{CIP} 1 / \mathrm{WAF} 1}$ results from the oxidative modification of $\mathrm{p} 53$ function? $\mathrm{p} 21^{\mathrm{CIP} 1 / \mathrm{WAF} 1}$ is a direct p53 target $[7,8]$. The latter itself is also regulated by redox modification through the thioredoxin family [12]. Reduced p53 has been reported to display higher DNA binding activity [12]. It is possible that a low level of oxidative stress induced by DEP leads to a more oxidised p53, which, in turn, results in decreased $\mathrm{p} 21^{\mathrm{CIP} 1 / \mathrm{WAF} 1}$ expression. A second question is whether the presence of DEP chemicals in serumfree conditions could provide mitogenic signals to the cell cycle machinery? Motorcycle exhaust particles, as well as fine PM, are able to activate the extracellular signal-regulated kinase (ERK) signalling pathway in human airway epithelial cells [13]. The activation of ERK has been shown to be necessary and sufficient to facilitate cell cycle progression [14]. DEP chemicals may act as a mitogen through the activation of ERK, which, in turn, leads to cell cycle progression. Thirdly, is the inhibition of $\mathrm{p} 21^{\mathrm{CIP} 1 / \mathrm{WAF} 1}$ by DEP a result of the activation of other gene products that inhibit $\mathrm{p} 21^{\mathrm{CIP} 1 / \mathrm{WAF} 1}$ ? One candidate for such gene products is c-myc [15]. Upon exposure to benzo[a]pyrene, a major DEP-associated PAH that is also present in cigarette smoke, human bronchial epithelial cells exhibit a dosedependent increase of c-myc expression [16]. c-myc is capable of blocking $\mathrm{p} 21^{\mathrm{CIP} 1 / \mathrm{WAF} 1}$ expression and epithelial growth arrest stimulated by transforming growth factor- $\beta 1$ [15]. Finally, it is important to recognise that A549 is a lung adenocarcinoma cell line, and it is known that cancer cells have altered cell cycle regulation and responses to redox disequilibrium [17]. Although A549 has been used extensively to test the toxicity of environmental toxicants, including DEP [18], its responses may not be representative of that from normal lung epithelial cells. It is imperative, therefore, to compare the responses of A549 with those of nontransformed lung epithelial cells.

Particulate matter, including diesel exhaust particle, contains a mixture of metals and organic chemicals. The ability of particulate matter to induce oxidative stress, as well as direct DNA modification, leads to a broad spectrum of biological responses. While the impact of particulate matter on inflammation and cytotoxicity has been extensively studied, its effect on oncogenes and tumour suppressor genes needs to be investigated in order to fully understand the mechanisms of particulate matter-related carcinogenesis.

\section{REFERENCES}

1 Dockery DW, Pope CA 3rd, Xu X, et al. An association between air pollution and mortality in six U.S. cities. $N$ Engl J Med 1993; 329: 1753-1759.

2 Vineis P, Husgafvel-Pursiainen K. Air pollution and cancer: biomarker studies in human populations. Carcinogenesis 2005; 26: 1846-1855.

3 Schuetzle D, Lee FS, Prater TJ. The identification of polynuclear aromatic hydrocarbon $(\mathrm{PAH})$ derivatives in mutagenic fractions of diesel particulate extracts. Int $J$ Environ Anal Chem 1981; 9: 93-144.

4 Risom L, Moller P, Loft S. Oxidative stress-induced DNA damage by particulate air pollution. Mutat Res 2005; 592: 119-137.

5 Pohjola SK, Lappi M, Honkanen M, Rantanen L, Savela K. DNA binding of polycyclic aromatic hydrocarbons in a human bronchial epithelial cell line treated with diesel and gasoline particulate extracts and benzo[a]pyrene. Mutagenesis 2003; 18: 429-438.

6 Ichinose T, Yajima Y, Nagashima M, Takenoshita S, Nagamachi $Y$, Sagai M. Lung carcinogenesis and formation of 8-hydroxy-deoxyguanosine in mice by diesel exhaust particles. Carcinogenesis 1997; 18: 185-192.

7 Clement A, Henrion-Caude A, Besnard V, Corroyer S. Role of cyclins in epithelial response to oxidants. Am J Respir Crit Care Med 2001; 164: Suppl. 10, S81-S84.

8 O'Reilly MA. Redox activation of p21Cip1/WAF1/Sdi1: a multifunctional regulator of cell survival and death. Antioxid Redox Signal 2005; 7: 108-118.

9 Corroyer S, Maitre B, Cazals V, Clement A. Altered regulation of G1 cyclins in oxidant-induced growth arrest of lung alveolar epithelial cells. Accumulation of inactive cyclin E-DCK2 complexes. J Biol Chem 1996; 271: 25117-25125.

10 O'Reilly MA, Staversky RJ, Watkins RH, Maniscalco WM. Accumulation of p21(Cip1/WAF1) during hyperoxic lung injury in mice. Am J Respir Cell Mol Biol 1998; 19: 777-785.

11 Bayram $H$, Ito $K$, Issa $R$, Ito $M$, Sukkar M, Chung K-F. Regulation of human lung epithelial numbers by diesel exhaust particles. Eur Respir J 2006; 27: 705-713.

$12 \mathrm{Hu}$ J, Ma X, Lindner DJ, et al. Modulation of p53 dependent gene expression and cell death through thioredoxinthioredoxin reductase by the Interferon-Retinoid combination. Oncogene 2001; 20: 4235-4248.

13 Lee CC, Cheng YW, Kang JJ. Motorcycle exhaust particles induce IL-8 production through NF-kappaB activation in human airway epithelial cells. J Toxicol Environ Health $A$ 2005; 68: 1537-1555.

14 Lents NH, Keenan SM, Bellone C, Baldassare JJ. Stimulation of the Raf/MEK/ERK cascade is necessary and sufficient for activation and Thr-160 phosphorylation of a nuclear-targeted CDK2. J Biol Chem 2002; 277: 47469-47475.

15 Pardali K, Kowanetz M, Heldin CH, Moustakas A. Smad pathway-specific transcriptional regulation of the cell cycle inhibitor p21(WAF1/Cip1). J Cell Physiol 2005; 204: 260-272.

16 Fields WR, Desiderio JG, Leonard RM, Burger EE, Brown BG, Doolittle DJ. Differential c-myc expression profiles in normal human bronchial epithelial cells following treatment with benzo[a]pyrene, benzo[a]pyrene-4,5 epoxide, and benzo[a]pyrene-7,8-9,10 diol epoxide. Mol Carcinog 2004; 40: 79-89.

17 Pani G, Bedogni B, Anzevino R, et al. Deregulated manganese superoxide dismutase expression and resistance to oxidative injury in p53-deficient cells. Cancer Res 2000; 60: 4654-4660.

18 Hukkanen J, Lassila A, Paivarinta $\mathrm{K}$, et al. Induction and regulation of xenobiotic-metabolizing cytochrome P450s in the human A549 lung adenocarcinoma cell line. Am J Respir Cell Mol Biol 2000; 22: 360-366. 